\title{
Development of the High Altitude Student Platform
}

\author{
T. G. Guzik, S. Besse, A. Calongne, A. Dominique, S. B. Ellison, R. Gould, D. Granger, D. Olano, D.
} Smith, M. Stewart and J. P. Wefel

Department of Physics and Astronomy, Louisiana State University, Baton Rouge, LA 70803, USA

\begin{abstract}
The High Altitude Student Platform (HASP) was originally conceived to provide student groups with access to the near-space environment for flight durations and experiment capabilities intermediate between what is possible with small sounding balloons and low Earth orbit rocket launches. HASP is designed to carry up to twelve student payloads to an altitude of about 36 kilometers with flight durations of 15 to 20 hours using a small zero-pressure polyethylene film balloon. This provides a flight capability that can be used to flight-test compact satellites, prototypes and other small payloads designed and built by students. HASP includes a standard mechanical, power and communication interface for the student payload to simplify integration and allows the payloads to be fully exercised. Over the last two years a partnership between the NASA Balloon Program Office (BPO), Columbia Scientific Balloon Facility (CSBF), Louisiana State University (LSU), the Louisiana Board of Regents (BoR), and the Louisiana Space Consortium (LaSPACE) has lead to the development, construction and, finally, the first flight of HASP with a complement of eight student payloads on September 4, 2006. Here we discuss the primary as-built HASP systems and features, the student payload interface, HASP performance during the first flight and plans for continuing HASP flights. The HASP project maintains a website at http://laspace.lsu.edu/hasp/ where flight application, interface documentation and status information can be obtained.
\end{abstract}

\section{Introduction}

A pressing issue in higher education is the need to attract and retain students in S\&E (science and engineering) curricula and to encourage these students to continue on with a career in a STEM (science, technology, engineering and math) related field. In the U.S., the national high technology workforce is rapidly aging, and the number of students entering the workforce as scientists and engineers has been steadily declining over the last several decades. This problem has been identified in numerous studies ranging from assessments of U.S. national security (Hart, Rudman, Armstrong, et al., 2001) to reports on the future of the U.S. aerospace industry (Walker, Peters, Aldrin, et al., 2002) and the Aldridge Commission's report on implementing the Vision for Space Exploration (Aldridge, Fiorina, Jackson, et al., 2004). For example, in the aerospace workplace close to $27 \%$ of workers will be eligible to retire by 2008 (Walker, Peters, Aldrin, et al., 2002). Across all science and engineering (S\&E) fields more than half of the degreed workers are aged 40 or older and retirements will dramatically increase over the near term (National Science Board, 2004).

Concern about the S\&E workforce is not surprising. A typical engineer or scientist is a highly trained, experienced individual who can call upon multiple practical skills, is familiar with teamwork, must effectively communicate results by writing numerous reports, documents and presentations and is intimately involved in the daily management of people, money and time. Yet many students entering a university have few practical skills, have problems with writing and "computer literacy" (limited to web usage), and are just beginning to understand the concept of time management. Bridging the gap between these two extremes at the university level has mostly been focused on providing content knowledge with little emphasis on how to integrate and apply this knowledge to real world problems. Some engineering departments include a "capstone" or design course in the last year, but most science departments have no such organized method. 
In the U.S., the National Space Grant Student Satellite Program has involved hundreds of students, at higher education institutions across the country, in the design, construction and operation of aerospace payloads ranging from simple balloon experiments to compact Earth-orbiting satellites. These students are helped to acquire practical skills in electronics, programming and design and led through a development life cycle, culminating in the exciting launch and flight operation of their payload. One end of this approach involves the students in the design and development of small payloads (less than about 500 grams) that can be carried up to high altitude (around 30 kilometers) by a latex sounding balloon, such as the LaACES program in Louisiana (Ellison, Giammanco, Guzik, et al., 2006). This approach has been very successful in helping students integrate their content knowledge with practical skills and to understand the end-to-end process of project development. Sounding balloons, however, are usually constrained in flight duration ( $\sim 30$ minutes above 24 kilometers) and payload weight, limiting the kinds investigations that are possible. Student built micro- or picosatellites can be placed in low Earth orbit removing the flight duration constraint, but there is significantly increased expense, scheduling difficulty that can carry across years as a rocket booster ride is negotiated and risk associated with a rocket launch into low Earth orbit.

An intermediate approach takes advantage of the mature technology and extensive body of experience in scientific ballooning available through professional scientific ballooning organizations such as the NASA Balloon Program Office (BPO) and Columbia Scientific Balloon Facility (CSBF). For multiple decades, the CSBF has flown payloads weighing up to several tons to altitudes up to 160,000 feet for durations of more than 40 days using zero-pressure polyethylene film balloons. Such capability far exceeds what is required for most student payloads, but an appropriately tailored inexpensive, reusable "ballooncraft" platform, can provide student payloads with extended durations in a nearspace environment while maintaining low cost and a regular schedule of "launches".

The High Altitude Student Platform (HASP) has adopted just this approach. HASP was developed by Louisiana State University (LSU) in partnership with the Louisiana Board of Regents, the Louisiana Space Consortium, NASA BPO and the CSBF to carry up to twelve student payloads at a time to an altitude of about 36 kilometers with flight durations of 15 to 20 hours using a small zeropressure polyethylene film balloon. This platform provides a flight capability greater than sounding balloons and can be used to flight-test compact satellites, prototypes and other small payloads designed and built by students.

The advantage of this approach is illustrated in Figure 1 which shows the flight profile of the first HASP flight (September 4, 2006) compared with that of a typical latex sounding balloon flight. Not only was HASP able to achieve more than 6 kilometers of altitude in addition to the peak of the sounding balloon flight, but remained at this altitude for 15 hours exposing the student payloads to both day and night time extremes. The student payload capacity of HASP is indicated in Figure 2 where up to eight small payloads (limited to a mass of about 1 $\mathrm{kg}$ ) are located on fiberglass "outrigger" braces and

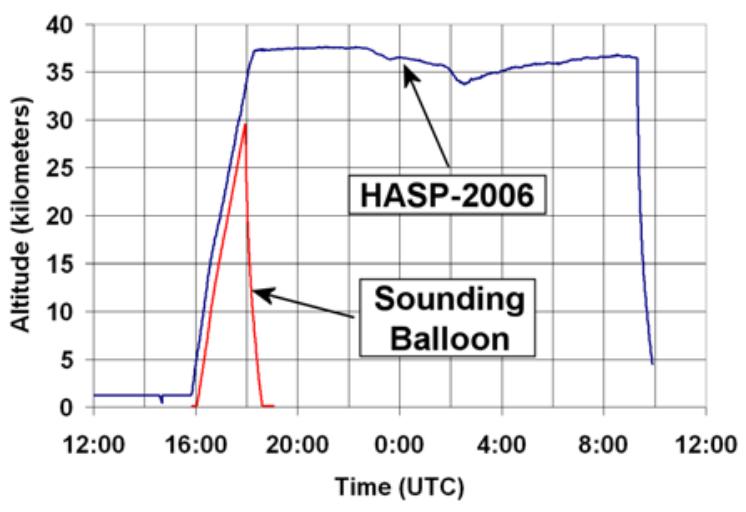

Fig. 1: Altitude vs. time flight profile for HASP compared to that of a sounding balloon flight.

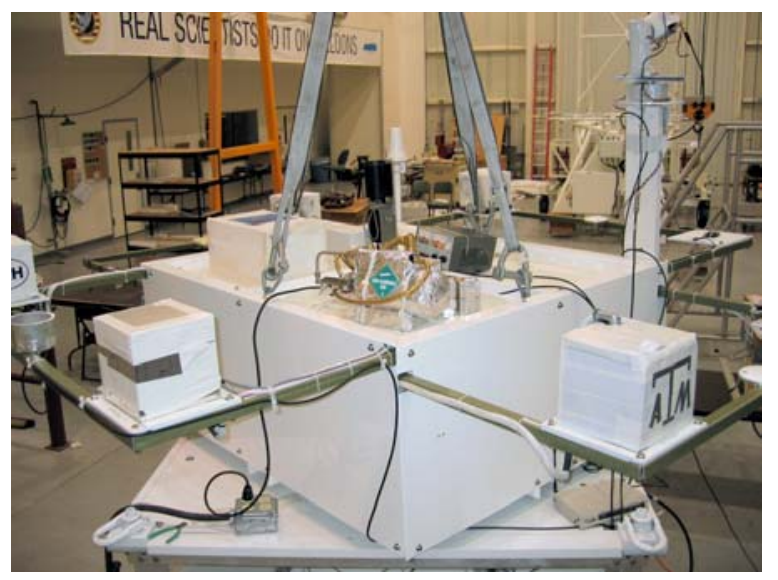

Fig. 2: Top view of HASP showing the small and large payload positions. 
up to four large payloads (mass limit of $\sim 10 \mathrm{~kg}$ ) are bolted to the top panels of the central frame. Each payload position is provided with 28 VDC power, a serial RS-232 communication link and auxiliary discrete and analog downlink lines. This kind of support, altitude and flight duration opens the door to more advanced and complicated student built experiments or detailed evaluation of the power, data acquisition and telemetry subsystems of student built satellite prototypes. In fact, while HASP is currently conceived for "short" 20 hour Continental US flights, a slightly modified version of HASP could easily be used for 15 - 30 day long duration balloon (LDB) or $~ 100$ day ultra long duration balloon (ULDB) flights.

\section{The HASP System}

During 2005 and 2006 the HASP system was designed, built, tested and achieved its first flight. Much of the HASP hardware and software is based on flight proven designs from the LSU Advanced Thin Ionization Calorimeter (ATIC) cosmic ray scientific investigation (Guzik, Adams, Ahn, et al., 1999; 2003). This has enabled us to minimize development costs and simplify operations.

\subsection{Configuration \& Structure}

Figure 3 is an image of the entire HASP flight configuration payload which is composed of two major components; the CSBF frame on the bottom and the HASP system on the top. The CSBF frame is the primary structural element of the payload and was recycled from an older test payload frame by the CSBF. This component houses all the subsystems CSBF requires to monitor and control the balloon vehicle. On the top corners of this frame are the attach points for cabling leading to the swivel, flight train, parachute and balloon. Each suspension cable is threaded through a PVC pipe increasing the rigidity of the suspension system and reducing the probability that, upon landing, the swivel would impact student payloads located on the top of HASP. On the bottom of the CSBF frame is the cardboard honeycomb crush pad and attach points for the ballast hopper suspension cables.

The interior of the frame supports a Mini-SIP (Support Instrument Package) that interfaces to the HASP electronics and provides uplink - downlink communication with the balloon payload. Usually ConUS (Continental U.S.) balloon payloads, such as HASP, use only a CIP (Consolidated Instrument Package) for vehicle control and communication, while a SIP is used for LDB payloads flown in Antarctica or from Sweden. As the HASP flight and ground software heritage flows from the ATIC LDB payload, use of a Mini-SIP in this instance (normally the Mini-SIP is used for ConUS flight testing of LDB payloads) significantly reduced the development cost and increased the reliability of the software. Another advantage of this decision is that at some future date HASP could be converted into an LDB payload, providing student payloads with 15 to 30 days of flight time, with minimum modifications to the flight systems. Finally, the frame interior also provided room for a variety of prototypes and test articles, allowing the CSBF to test fly new technology that may improve the performance of future professional scientific balloon systems.

HASP is located on the top of the CSBF frame and provides the interface for the student

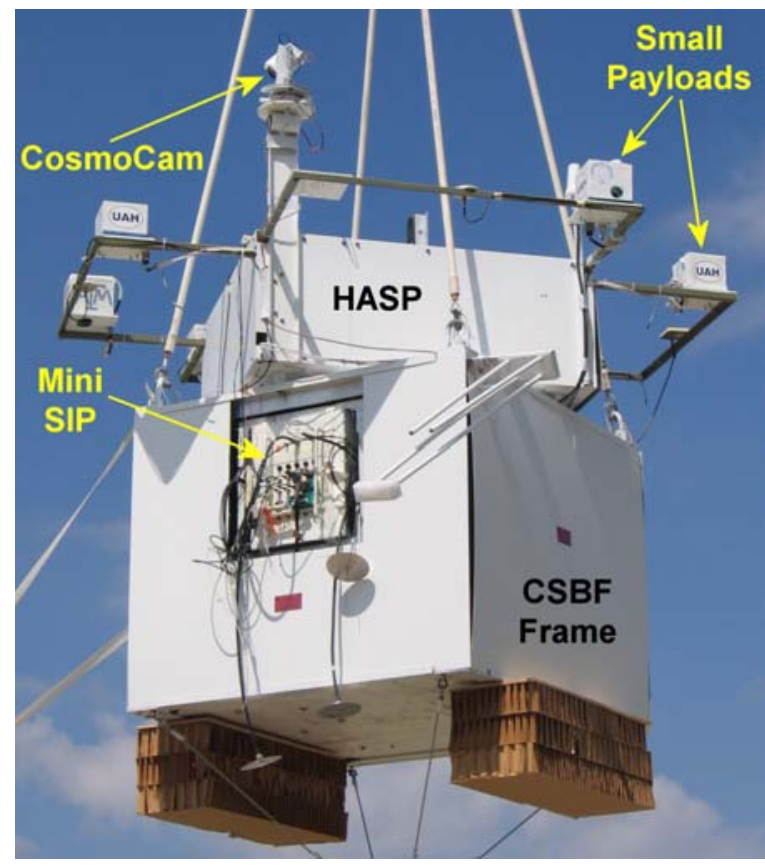

Fig. 3: The HASP payload as configured for the 2006 flight. 
payloads. The core structure of HASP is a welded aluminum gondola frame that is powder coated white with dimensions of $112 \mathrm{~cm}$ long, $91.5 \mathrm{~cm}$ wide, and $51 \mathrm{~cm}$ tall. On each side of the frame is bolted a thin aluminum sheet painted white on the outside and bare metal on the inside. These "solar shields" can be removed to provide access to the HASP internals. A radiation barrier of bare aluminum sheet is also attached to the frame interior bottom and top surfaces. Any exposed openings on the HASP top are covered by white painted PVC plates, again to act as solar shields. Attached to the core structural frame are four fiberglass frames that support the small $(<\sim 1 \mathrm{~kg})$ student payloads. Each brace extends $56 \mathrm{~cm}$ away from the aluminum frame and supports two student payload mounting plates, each of which includes standard mechanical, power and serial communication interfaces. One such brace is attached to each side of the core structure to accommodate up to eight student payloads. This configuration was chosen to minimize interference between the metal frame and any student payloads that may exercise their transmitters during flight. In addition, up to four large $(<\sim 10 \mathrm{~kg})$ student payloads can be mounted on the top of the core structure and have a power and communication interface similar to that of the small payloads. The top down view of HASP in Figure 2 shows the two large payloads and several small payloads flown during 2006. Finally, a vertical beam provides support for the CosmoCam real-time video camera provided by Rocket Science Inc. and a collection of GPS antennas are mounted on the fiberglass frames.

The mass budget for HASP as measured for the 2006 flight is given in Table 1. The total weight for HASP is 190.4 kilograms which includes the HASP frame, electronics, batteries, CosmoCam and student payloads. The CSBF frame including the Mini-SIP, batteries and test articles weighed 265.9 kilograms. Adding the parachute, flight train, terminate package, crush pad and ballast brings the total weight of the payload to 909.1 kilograms. Given this payload weight $\mathrm{a} \sim 311,500 \mathrm{~m}^{3}\left(11,000,000 \mathrm{ft}^{3}\right)$ volume zero-pressure balloon was chosen for flight.

\begin{tabular}{|c|c|}
\hline Component & Weight (kg) \\
\hline HASP System & 108.2 \\
\hline CosmoCam & 10.0 \\
\hline HASP Batteries & 43.6 \\
\hline Student Payloads & 28.6 \\
\hline Mini-SIP \& Batteries & 110.9 \\
\hline Mini-SIP Frame & 113.6 \\
\hline CSBF Test Articles & 41.4 \\
\hline Parachute, Train, UTP, Pad & 207.3 \\
\hline Ballast & 245.5 \\
\hline Total & 909.1 \\
\hline
\end{tabular}

\subsection{Command \& Control}

The HASP command and control subsystem, illustrated in Figure 4, provides the means for receiving and processing uplinked commands, acquiring and archiving the payload data, downlinking status information and interfacing with the student payloads. There are three primary control modules in the subsystem that communicate with each other over a $100 \mathrm{Mbps}$ (megabit per second) internal Ethernet network. These modules are the Flight Control Unit (FCU), the Data Archive Unit (DAU) and the Serial Control Unit (SCU). All modules run the QNX real-time operating system, which is POSIX complaint and facilitates programming across a distributed environment. Flight software processes on different modules communicate across an internal payload ethernet using a client / server message passing scheme. The software is event driven where the "events" are classified as experiment triggers, timeouts, commands and messages. The HASP flight software evolved from that developed for the ATIC experiment with a few additions such as the serial communication processes that are needed for communication with the student payloads.

For HASP, the different modules can be run independently and each is assigned a set of specific tasks. The FCU "manages" the subsystem; decoding commands received from the CSBF supplied MiniSIP and distributing them, watching for units that may need to be reset, collecting status information and downlinking data through the Mini-SIP. The downlinked telemetry through the Mini-SIP has a maximum rate of $38 \mathrm{kbps}$. In addition, the FCU also monitors the voltages and currents of the power system and collects environmental temperature information for housekeeping records. Without the FCU, 


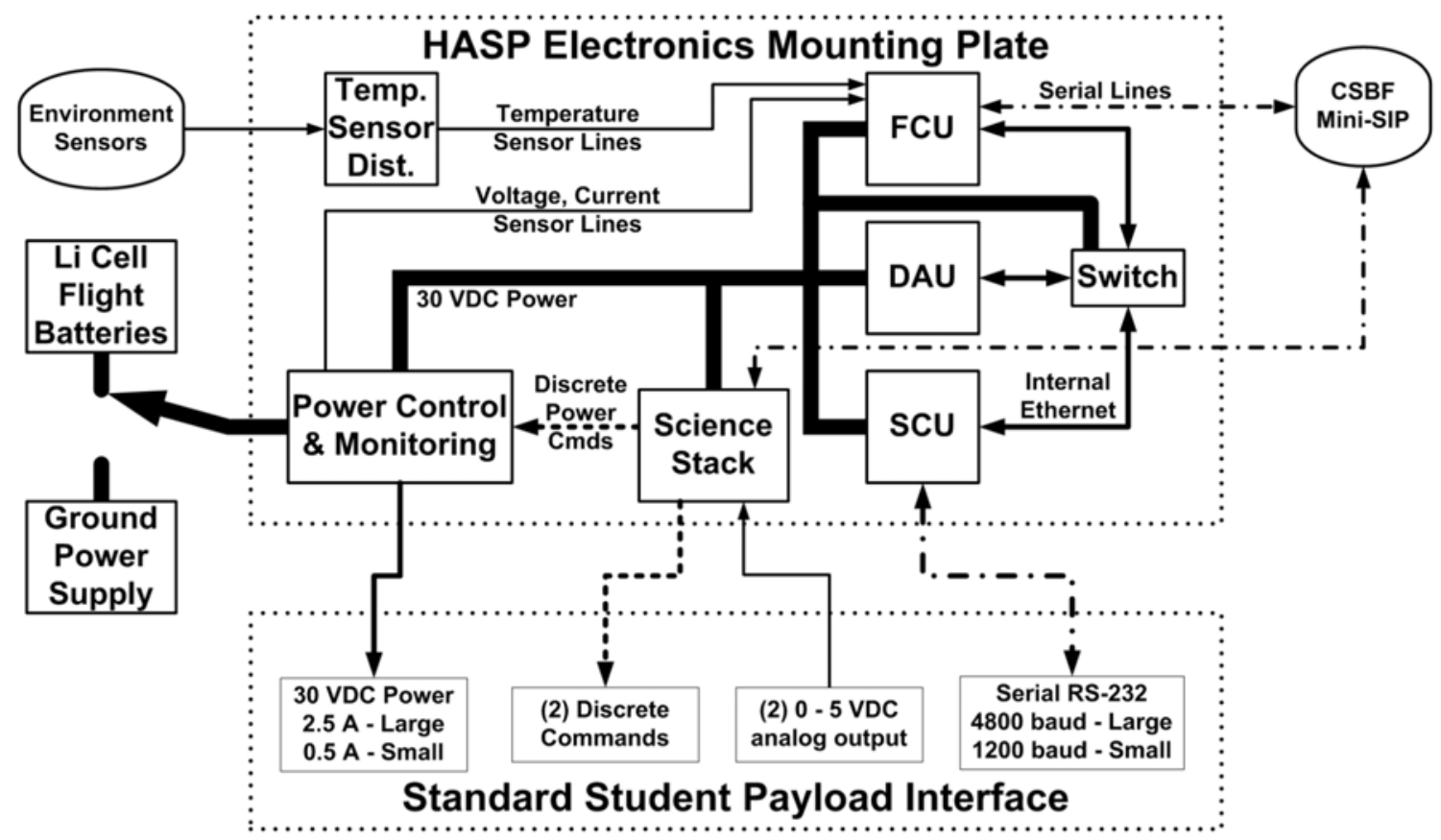

Fig. 4: HASP flight electronics system and student payload interface.

communication with the ground is lost, but this does not mean complete system failure as data would still be recorded on-board. The DAU controls the on-board recording of all data, making these data records available to other processes on the network, controlling the HASP GPS receiver and managing the timestamping of all records. All on-board recording is to Compact Flash cards which appear as hard disks to the operating system, but function well in hard vacuum. If the DAU and only the DAU goes down, data is re-routed to the FCU for direct data downlink without buffering and storage. Therefore, if the FCU and telemetry is functioning, all data can be retrieved on the ground. The Serial Communications Unit (SCU) runs the software which communicates with the student payloads. Without this unit, student data will not be collected.

The ground software consists of several different independent processes. The Housekeeping graphical user interface (GUI) displays all temperature, voltage and current data collected on board HASP. It is updated each time a housekeeping record is received. This period can be adjusted from 1 second to many hours. The Student Payload Serial Interface GUI or SPSI GUI displays the status of the data transfer from all student payloads to the ground. It also writes all student data to files that are retrieved by the student groups via the HASP web-server. The number of files written, the name of the current file being written to, the number of bytes collected are some of the status indicators displayed by the SPSI GUI. The Error and Status display is a very important part of determining the overall health of the experiment. Each time an error or status record is received, it is added at the top of a list in the display as a single line.

The command display interfaces with the CSBF ground telemetry equipment and allows commands to be transmitted to HASP. Each of the ground processes can be run in one of three autodetected and set modes. For ground calibration and testing, each of the ground displays can be run with their corresponding flight process. In this mode the display connects directly to the flight process. A network connection between the ground and flight computer is necessary. For flight and ground system testing without telemetry, all ground processes can be run with all flight software. In this mode, the ground display connects to the flight buffer process group (BPG) to retrieve records. This mode also requires a network connection. The ground system displays can also be run in full flight mode (includes telemetry). In this mode the GUI connects to the ground data buffer to retrieve all records. 
All module stacks are built from commercially available PC/104 CPU, I/O, and peripheral control cards. Each stack uses a Microcomputer Systems, Inc. MSI-CM587 that integrates a NS Geode GX1-300 MHz processor with Ethernet, IDE, SVGA/LCD, keyboard, mouse, USB, serial, parallel and CompactFlash interfaces on a single PC/104 form factor board. This low power single board computer card allowed us to reduce the size of the HASP stacks by eliminating peripheral cards (e.g. Ethernet, VGA) that would be needed to support older CPUs. In addition, the FCU includes a MSI-DMP1800 four port RS232 serial card for communication with the Mini-SIP, two MSI-P412 cards each of which contains 32 channels

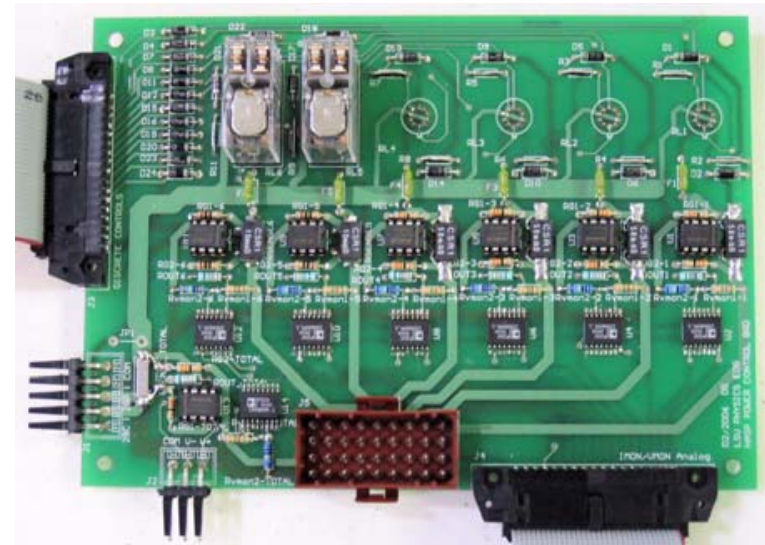

Fig. 5: HASP power relay and monitoring board custom designed and built at LSU. of 12-bit ADCs that are used to digitize the voltage, current and temperature environment data, plus a MSI-NC0072 to convert our 28 VDC power bus to the voltages required by the stack. (The NC0072 also includes 24 digitial I/O lines that are not used by HASP at this time.) The SCU stack is composed of a CM587, a NC0072 and three DMP1800 to support serial communications to all student payloads. Finally, the DAU includes a CM587, a NC0072 plus a MSI-P602 GPS card which Microcomputer Systems, at our request, configured to use the Trimble Lassen iQ 12 channel GPS engine. To prevent commercial grade GPS units from being used for military applications the units are designed to fail under certain conditions; usually when the unit exceeds some combination of altitude and/or velocity. However, identifying a GPS unit that remains functional at balloon altitudes can, at times, be frustrating as the modes and conditions of failure are not always well documented by the manufacturer. After some datasheet research, discussion and testing, we chose the Trimble Lassen iQ as it has a one PPS (pulse per second) output plus an operational limit determined by altitude $(<18 \mathrm{~km})$ AND velocity $(<515 \mathrm{~m} / \mathrm{s})$. Thus, given that even fast balloon velocities are very low $(<20 \mathrm{~m} / \mathrm{s})$, this unit will remain functional up to the expected altitude of $37 \mathrm{~km}$ or so. The P602 on the DAU provided the HASP latitude, longitude and altitude position information during the flight and the one PPS signal was used to keep the clocks on all three stacks synchronized to well within 500 microseconds.

Temperature sensors are placed at strategic locations around HASP (e.g. batteries, solar shield panels, exterior) to monitor the environment and assess the thermal performance of the system. Cabling from these 28 sensors is initially routed to a custom temperature sensor distribution board and then to the P412 boards in the FCU. The CSBF supplied Science Stack provides a separate line of communication, through the Mini-SIP, between HASP and the ground that is independent of the FCU. For the recent HASP flight the Science Stack was configured with two command modules and one ADC module. The ADC module provides student payloads with a simple mechanism for downlinking a pair of $0-5$ VDC channels for monitoring some aspect of their experiment. The command modules are used to control specific or "discrete" events in the payloads. Such discrete events could be to unlatch an optics cover or deploy an antenna. In HASP such discrete events are used to control the on and off states within the power system.

\subsection{Power System}

The input power source for HASP is about 30 VDC and is provided by either a Lambda ZUP3624 for ground operations or eight B7901-11 eleven cell lithium sulphur dioxide batteries for flight. The Lambda power supply can be easily run from a generator on the balloon launch vehicle and swapped out for the flight batteries about an hour before launch. Two of the flight batteries are dedicated exclusively to the HASP control systems while the remaining six provide power to the student payloads. Input power is routed initially to two master control relays; one for the HASP control system and one for the student 
Table 2: HASP-06 Power Budget

\begin{tabular}{cccc}
\hline Component & Volts & mA & Watts \\
\hline FCU & 30 & 340 & 10.2 \\
\hline DAU & 30 & 250 & 7.5 \\
\hline SCU & 30 & 380 & 11.4 \\
\hline Other HASP & 30 & 125 & 3.8 \\
\hline CosmoCam & 30 & 190 & 5.7 \\
\hline $\begin{array}{c}\text { Student } \\
\text { Payloads }\end{array}$ & 30 & 920 & 27.6 \\
\hline Total & & & $\mathbf{6 6 . 2}$
\end{tabular}

Science Stack, one for on and one for off, control each relay and another set of discrete commands control the system and payload master relays. Power is distributed throughout HASP as 30 VDC and converted locally as needed, which has the advantage of significantly simplifying the payload power system.

The power budget measured during the 2006 HASP flight is shown in Table 2. The currents presented in the table are averaged over the time that HASP was at float and the particular component was powered on. From this table we see that the HASP systems draw about 1.1 amps at 30 VDC, while the student payloads and CosmoCam account for about another 1.1 amps. Each B7901-11 battery is rated to provide about $34 \mathrm{Ahr}$ at $20^{\circ} \mathrm{C}$ and about $19 \mathrm{Ahr}$ at $-20^{\circ} \mathrm{C}$. Thus, the two batteries dedicated to the HASP system should be able to supply power for more than 60 hours if the batteries remain at room temperature or at least 35 hours if we de-rate the batteries for the colder temperature. Likewise the six "payload" supply batteries would be able to power the student payloads and CosmoCam from 100 hours (cold) to more than 180 hours (warm). The student payload load during the 2006 flight is very light compared to the maximum $(\sim 14 \mathrm{~A})$ that HASP is designed to handle. For this maximum current draw the six payload batteries would be able to supply power for about 15 hours (warm case), which is somewhat short of the nominal 20 hour operational lifetime desired. Adding another four batteries would bring this lifetime up to about 24 hours. The student payload current requirement will be evaluated for each flight and batteries will be added or removed as required.

\subsection{The HASP Electronics Mounting Plate}

One of the major innovations incorporated into HASP was to mount all the command and control components (i.e. upper dotted box in Figure 4) on a single $109 \mathrm{~cm}$ by $36 \mathrm{~cm}$ Electronics Mounting Plate (EMP). This has the primary advantage that the EMP can be easily removed from the HASP frame for easy access to all the electronics during debugging, testing and/or servicing. In addition, a complete flight spare EMP was built during HASP development and can be used as a "plug and play" replacement in the field should problems arise with the original. This helps improve the overall reliability of HASP, reducing the time necessary to bring HASP to flight ready status and minimizing flight operation costs.

Figure 6 shows an EMP on a desktop undergoing servicing after being removed from the HASP frame. The white boxes on the right hand side of the plate are the $\mathrm{PC} / 104$ computer stacks and from right to left include the FCU, SCU and DAU. Immediately to the left of the DAU is the internal

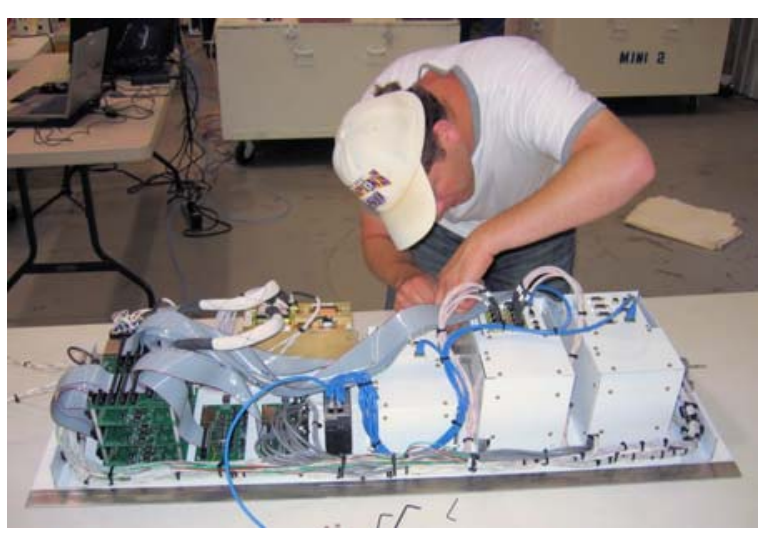

Fig. 6: Desktop servicing of the HASP electronics mounting plate. 
Ethernet switch and the two temperature sensor distribution boards. Above the switch is the gold (non-flight) science stack and to the left of the temperature boards is the stack of power system relay boards. All internal wiring is attached to the EMP and connectors for interfacing with the student payloads are on patch panels on each end of the plate.

Figure 7 shows the EMP being slid on its guide rails into the HASP frame. After the plate is positioned it is pinned in place to prevent any lateral movement. The wiring harness to the student payloads then plugs into the connectors on the EMP patch panel, one of which is visible in the figure. Finally, temperature sensors cables are plugged into the temperature distribution board and power is supplied through a connector on one

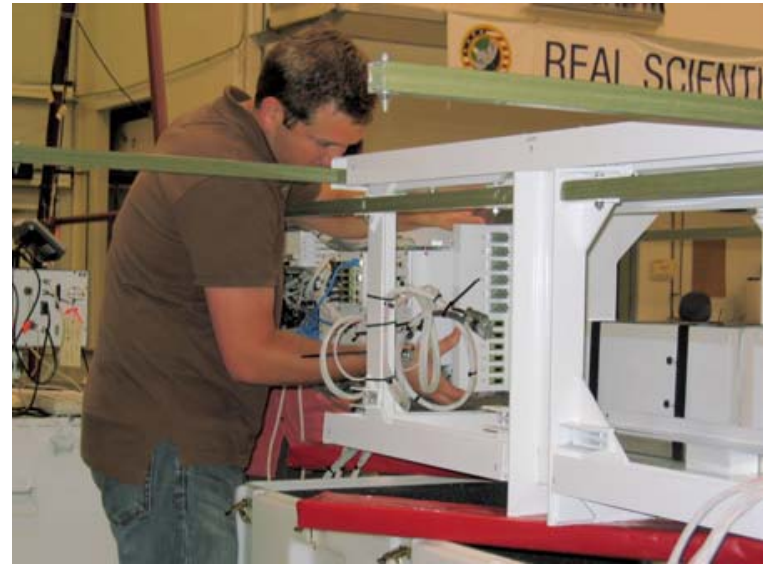

Fig. 7: The entire HASP electronics mounting plate can be easily slid in and out of the structural frame as shown here. of the patch panels. Replacing the entire EMP is literally a less than 5 minute operation.

\subsection{The HASP - Student Payload Interface}

HASP provides a power, and communication interface to each student payload to enable each experiment to the controlled and monitored during the flight. The functional details of this interface are shown in the lower dotted box of Figure 4. Two payload classes, large and small, with somewhat different functional capability are supported by HASP. Large payloads are restricted in mass to less than about $10 \mathrm{~kg}$ and are located on the top of the HASP frame. Four payload positions are possible for large payloads. Small payloads must mass less than about $1 \mathrm{~kg}$ and eight positions are possible on the ends of the fiberglass braces (see Figures 2 and 3)

Serial communication uses the RS-232 standard and is provided to both classes of payloads through a DB9 connector. Student payloads can transmit their data as a "stream" over this link at speeds up to 4800 baud for large payloads and 1200 baud for small payloads. This stream is packaged into 1400 byte records by HASP and archived on-board by the DAU as well as transmitted to the ground. Once on the ground, data is written to files named according to the student payload identification number from which it came and the day, hour, minute and second on which the file was opened. The data is written to these files as it was received from the student payload. An open file will be closed and a new file will be opened each time one hundred records (i.e. 140,000 bytes) have been written to the current open file. Thus, for the maximum transmission rate, a new file will be opened every $\sim 20$ minutes for a small payload and every $\sim 5$ minutes for a large payload. The new files are transmitted over the internet from the flight line ground system to LSU where they are then made available on the HASP website. In this fashion, students at their home institution can monitor their experiment in near real time.

The serial communication line can also be used to uplink two byte commands to the student payload. These commands are transmitted by the HASP operator on the flight line upon request by the student team leader from a list provided to the operator prior to flight. The operator includes the payload identification (ID) number with the command and transmits it to HASP. Once the command is received onboard, HASP formats the command and routes it to the particular serial link determined by the payload ID. It is the responsibility of the student payload to receive the command, unpack the format and validate the command prior to execution. While, in principle, all 16 bits could be used to identify different payload commands, in practice, we usually recommend that the first byte be used for command validation. This still leaves one full byte or 256 different possibilities for changing the state of the experiment during flight. 
Power, discrete commands and analog output are provided to the student payload on a twenty pin EDAC 516 connector. Power is delivered as $+30 \mathrm{VDC}$ on four pins of the connector and a second set of four pins is the power ground. Small payloads are allowed to have a maximum current draw of about 500 milliamps, while the current limit for large payloads is increased to about 2.5 amps. There are two analog output channels on the EDAC 516 connector each of which will accept a signal in the 0 to $5 \mathrm{VDC}$ range. These channels are sampled by the Science Stack once a minute, digitized and transmitted by the CSBF systems to provide real-time monitoring of two key payload parameters (e.g. temperatures). In addition, two pins on the connector are dedicated to discrete commands provided by the CSBF Science Stack as opencollector outputs that can sink a maximum of 200

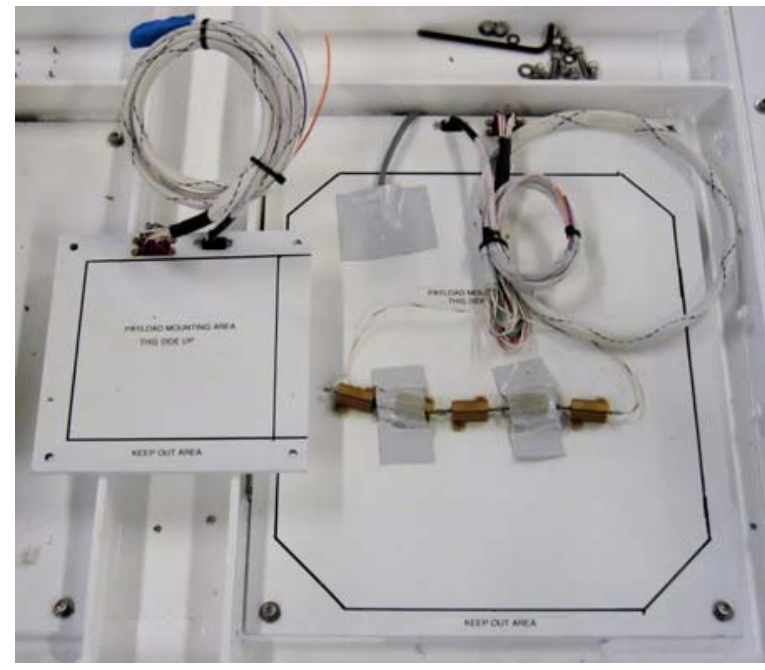

Fig. 8: The small (left) and large (right) student payload mounting plates. milliamps at a maximum of 50 volts. The open collector output is pulled low for 100 milliseconds when a command is sent. Discrete commands are used to change the state of the payload in some fashion such as opening a mirror cover or deploying a boom. Payload power on and power off is already controlled by discrete command through the HASP power system.

The mechanical interface between HASP and a student payload is handled by providing each team with a standardized mounting plate as shown in Figure 8 for both small and large payloads. Each plate is constructed of $1 / 4$ " PVC and is marked to indicate the area which can be modified by the student team to support their payload structure. Small payloads are allowed a footprint of $15 \mathrm{~cm}$ by $15 \mathrm{~cm}$ while large payloads must conform to a footprint of less than $38 \mathrm{~cm}$ by $30 \mathrm{~cm}$. (Both classes of payloads must be less than $30 \mathrm{~cm}$ tall.) Exterior to these footprints is HASP structure and mounting bolt holes and can not be modified or intruded upon by the student payload. Each plate comes equipped with the DB9 and EDAC 516 interface connectors plus a wiring pigtail for direct connection to terminals in the student payload. Once a student team is accepted for flight they are shipped the appropriate size mounting plate plus a document that details the electrical, mechanical and communication protocols of the HASP student payload interface. This allows each team to build their payload, while assuring compatibility with the HASP systems.

\section{The First Flight of HASP}

Design and development of the HASP system began during fall 2005 with support from the Louisiana Board of Regents, the LSU Department of Physics \& Astronomy, the LSU College of Basic Science and the Louisiana Space Consortium. An initial version of HASP was completed by April 2006 and taken to the CSBF for thermal testing. This test was used to thermal stress the flight systems for extreme cold $\left(-50^{\circ} \mathrm{C}\right)$ and hot $\left(+50^{\circ} \mathrm{C}\right)$ cases plus measure the interior temperatures relative to the outer solar shields. We found that the HASP electronics operated with no problems during these temperature extremes and the relative temperature data was used with a steady-state thermal model to estimate flight performance. This trip also allowed

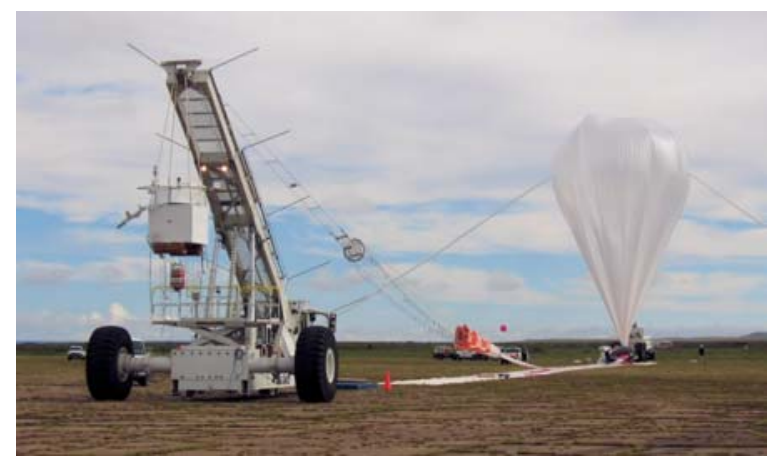

Fig. 9: HASP payload and balloon being readied for launch on Sept. 4, 2006 
us to evaluate operating HASP in the field with a two person crew and to identify any changes for the final version. These changes were relatively few and were completed during June 2006 in time for student payload integration in July.

In parallel to the HASP development we released a "Call for Payloads" (CFP) document in November 2005. The CFP contained a description of HASP, details about the student payload interface and instructions for how to apply for a payload seat. By the time of the application deadline in March 2006 we had received applications for nine payloads from four institutions and decided to accept all of them for the 2006 flight. Subsequently, two payloads dropped out and one of the institutions added another payload resulting in eight payloads that were eventually flown on HASP. These payloads were integrated with HASP at LSU in late July, during which the mechanical, power and communication interfaces were verified and plans for flight line operations were discussed. Most of the student payloads had either some interface issue to resolve or some refinement they wanted to add and, consequently, chose to deliver their payload to the flightline themselves.

Following initial student payload integration in early August 2006, HASP was packed and shipped to Ft. Sumner, New Mexico for flight operations. The two person HASP flight crew arrived in Ft. Sumner at the beginning of the third week in August and subsequent setup went very smoothly. Student team members also arrived during the week to deliver and integrate their payload. HASP was integrated with the CSBF frame and Mini-SIP and was ready for compatibility testing by the following week. By August 29, 2006 HASP was declared fully flight ready. Now it was merely a matter of waiting for the proper launch and high altitude wind conditions. Unfortunately classes had started at most institutions by this time and the students were unable to wait to see the launch. However, several years ago CSBF implemented a series of launch site webcams for each of its primary operational field sites and these, coupled with the CosmoCam webcam on board HASP, allowed the students to view launch and flight operations from their home institution.

Finally, on September 4, 2006 weather conditions appeared promising and the HASP payload was picked up by the launch vehicle and rolled out to the launch pad. Shortly after dawn the parachute and 311,500 cubic meter balloon were attached to the payload. Just before balloon inflation was scheduled to begin, HASP was switched to battery power and all payloads were powered up for flight. Balloon inflation took about 40 minutes to complete and Figure 9 shows HASP on the launch vehicle with the almost fully inflated balloon in the background. HASP was launched at 15:51 UTC and reached float at about 18:18 UTC. As seen in Figure 1, HASP remained at an average float altitude of 36.3 kilometer (maximum $37.7 \mathrm{~km}$, minimum $33.7 \mathrm{~km}$ ) for 15 hours and was terminated on September 5, 2006 at 09:18 UTC. Payload landing was just southeast of the Grand Canyon in Arizona at 10:02 UTC.

The thermal testing and modeling done earlier in the year indicated that HASP should perform well in the high altitude near-space environment and in-situ measurements during the flight confirmed this expectation. Figure 10 shows

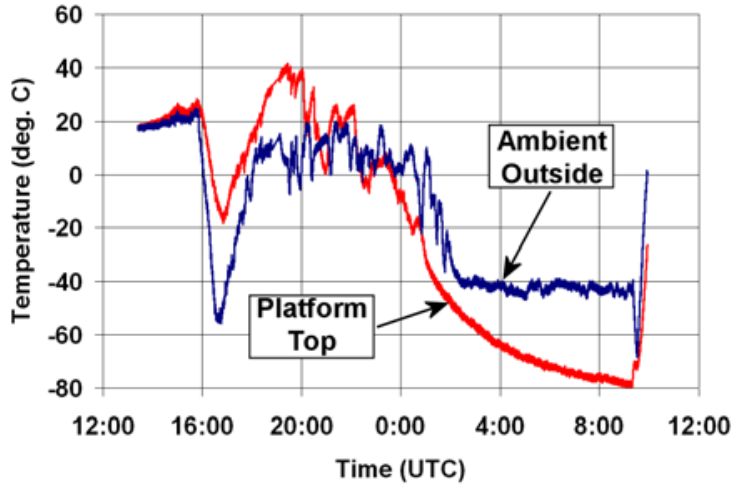

Fig. 10: Characteristic temperatures outside HASP during the 2006 flight.

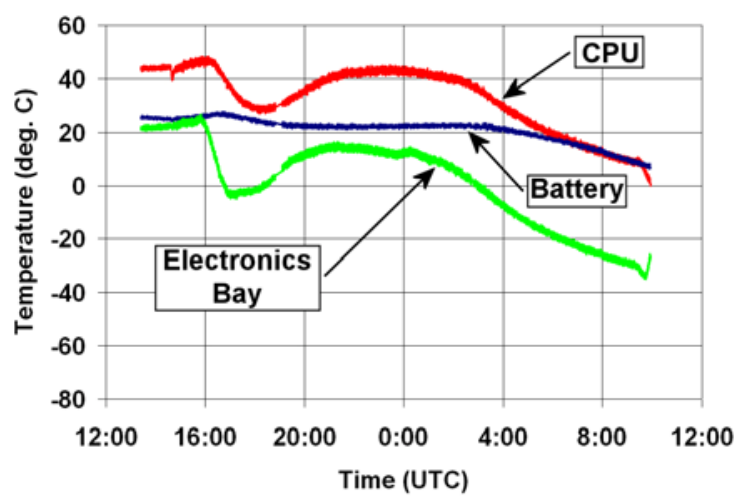

Fig. 11: Characteristic temperatures inside HASP during the 2006 flight. 
temperature measurements taken during the HASP flight from two sensors located external to HASP. The "Platform Top" sensor (red curve) was placed in the center of one of the unoccupied large student payload plates on the top of the HASP frame. From this location the sensor could "see" the sky and heat leaking from the HASP interior, but was shielded from Earth radiation. The "Ambient Outside" sensor (blue curve) was suspended about $5 \mathrm{~cm}$ below an unoccupied small student payload seat at the end of one of the fiberglass braces. In this position the sensor was well isolated from heat conducted through the HASP structure and had field of views to both the sky and Earth. Both curves show the typical atmospheric temperature profile as the balloon gains altitude; an initial decrease to a minimum at the tropopause followed by an increase until float altitude is reached. Once at float, the "ambient" sensor reports temperatures in the range $0^{\circ} \mathrm{C}$ to $20^{\circ} \mathrm{C}$ with variations presumably due to payload oscillations alternately shading and lighting the sensor. This behavior continues until about 9/5/06 00:00 UTC when the temperature begins to drop as the Solar elevation decreased. For the balloon position and altitude, local sunset was estimated to occur at about 9/5/06 02:00 UTC after which the "ambient" sensor remains at a steady $-40^{\circ} \mathrm{C}$ until termination at 09:18 UTC, presumably due to infrared emission from Earth. Following termination the sensor again shows the atmospheric temperature profile. Temperature readings from the "top" sensor are influenced primarily by the solar elevation angle and heat conducted through the HASP structure. Thus, the temperature peaks at local noon around 9/4/06 19:00 UTC and decreases to sunset. After sunset the temperature continues to decrease as heat slowly leaks from the HASP interior and there is little input from Earth IR.

Several temperatures reported by sensors interior to HASP are shown in Figure 11. The "CPU" sensor (red curve) provides a direct measurement of the FCU central processor temperature, the "Battery" temperature (blue curve) is an average of four sensors located within the lithium cell battery packs and the "Electronics Bay" sensor (green curve) is suspended about $5 \mathrm{~cm}$ below the top of the HASP interior between the Electronics Mounting Plate and the solar shield. All three curves vary smoothly throughout the flight reflecting the relative thermal isolation between the interior and the exterior. The "bay" temperature stays in the range $\sim 0^{\circ} \mathrm{C}$ to $\sim 15^{\circ} \mathrm{C}$ until after sunset when it drops to a bit more than $-30^{\circ} \mathrm{C}$. The CPU temperature remains well within operating range throughout the flight with a maximum less than $45^{\circ} \mathrm{C}$ and a minimum at the end of the flight above $0^{\circ} \mathrm{C}$. Finally, the "battery" temperature was remarkably steady in the range $20^{\circ} \mathrm{C}$ to $26^{\circ} \mathrm{C}$ until several hours after sunset when the temperature decreased to about $6^{\circ} \mathrm{C}$ just before landing. This excellent performance has implications for the next HASP flight. Since we can be assured of maintaining "warm" batteries, we can size the number of cells to fit the student payload current load (see section 2.3).

All HASP systems performed flawlessly during the flight. After initial flight configuration setup HASP required no further resets and no system reboots were required. The only commands sent during the flight were to power off the student payloads just before termination. HASP transmitted a continuous stream of diagnostic records, on-board data storage status messages, temperature, voltage and current information, student payload serial data, as well as GPS position, altitude and time data. These data were downlinked to the HASP ground station for real-time monitoring and then transmitted over the internet to

Table 3: Summary of HASP-06 Student Payloads

\begin{tabular}{ccccc}
\hline Payload & Institution & $\begin{array}{c}\text { Power } \\
(\mathbf{m A})\end{array}$ & $\begin{array}{c}\text { Weight } \\
(\mathbf{k g})\end{array}$ & Investigation \\
\hline ITBE-1 & UAH & 115 & 1.2 & Infrared sensing of balloon temperature \\
\hline GeoCam & TAMU & 380 & 1.6 & Remote sensing from high altitude \\
\hline AINS & LSU & 60 & 0.6 & Accelerometer inertial navigation sys. \\
\hline UAH-Cam & UAH & 60 & 1.0 & Digital camera imager \\
\hline ITBE-2 & UAH & 100 & 1.1 & Infrared sensing of balloon temperature \\
\hline ITBE-3 & UAH & 110 & 1.3 & Infrared sensing of balloon temperature \\
\hline CRS & ULL & n/a & 11.8 & Cosmic ray nuclear emulsion stack \\
\hline Aerospike & LSU & 95 & $\sim 10.0$ & Rocket nozzle performance vs altitude \\
\hline
\end{tabular}




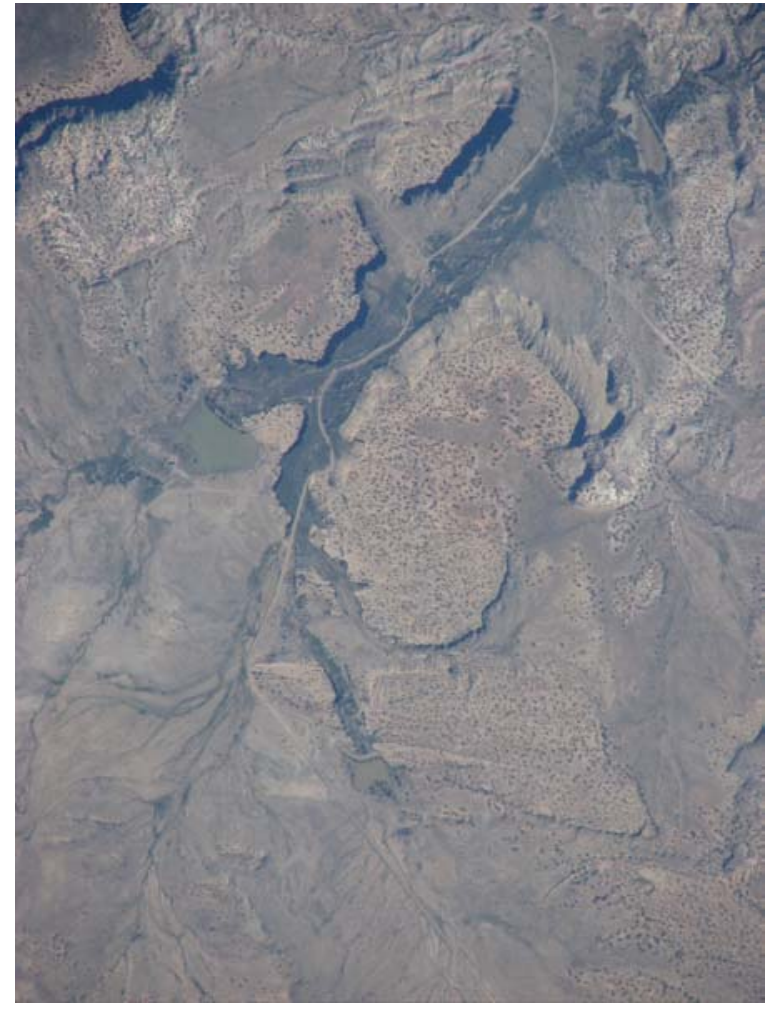

Fig. 12: View from 37 kilometer altitude as provided by the GeoCam student payload during the 2006 HASP flight.
LSU where they were made available in near realtime on the HASP website. A webmap was also included on the HASP site where the GPS position information was plotted to provide tracking of the payload. In this fashion students could monitor the flight and their payload data from their home institution.

The student payloads flown during this first HASP flight are summarized in Table 3 which lists the payload name, institution, power, weight and a brief description of the payload investigation. The Infrared Thermal Balloon Experiment (ITBE) of the University of Alabama - Huntsville (UAH) included three infrared telescopes located at different seats on the HASP braces looking up at the balloon to measure the thermal characteristics throughout the flight. UAH also included a separate camera payload to attempt to image the balloon as well as the horizon. The GeoCam payload developed by students at Texas A \& M University (TAMU) was a lookdown camera designed to study remote sensing techniques. During the flight GeoCam collected 1650 images and one of these remarkable pictures is shown in Figure 12. The TAMU team is in the process of stitching together their images into panoramas and making these available on their website (http://sei.tamu.edu/geocam/). The

University of Louisiana - Lafayette developed a nuclear emulsion stack experiment designed to student high energy cosmic rays. Finally, LSU students provided two experiments. The Accelerometer Inertial Navigation System (AINS) from LSU Physics \& Astronomy uses three precision accelerometers to measure the motion of the payload, which consequently can be integrated to derive orientation and position. In addition, LSU Mechanical Engineering students developed the Aerospike experiment to study the performance of a conventional and an aerospike rocket nozzle as a function of altitude. The Aerospike payload is shown in Figure 13 with the cold gas pressure vessel in the foreground and the aluminum block rocket nozzle models on the right hand side. The power consumption listed in Table 3 for each payload is a time average of their individual current draw over the period they were operational during the flight. The CRS experiment was passive and, consequently, had no current draw. The payload weights are all measured values with the exception of the estimate for the Aerospike experiment. As of this writing all student teams are in the process of analyzing their flight data with preliminary assessments available soon.

Finally, HASP was able to partner with Rocket Science, Inc. to include the CosmoCam web camera during the flight. This device is designed to bring live views of the balloon launch and flight from on-board the payload to students, classroom and the general public. The video camera head, mounted on a vertical stanchion (see Figure 3), includes a $26 \mathrm{x}$ optical zoom with full pan and tilt

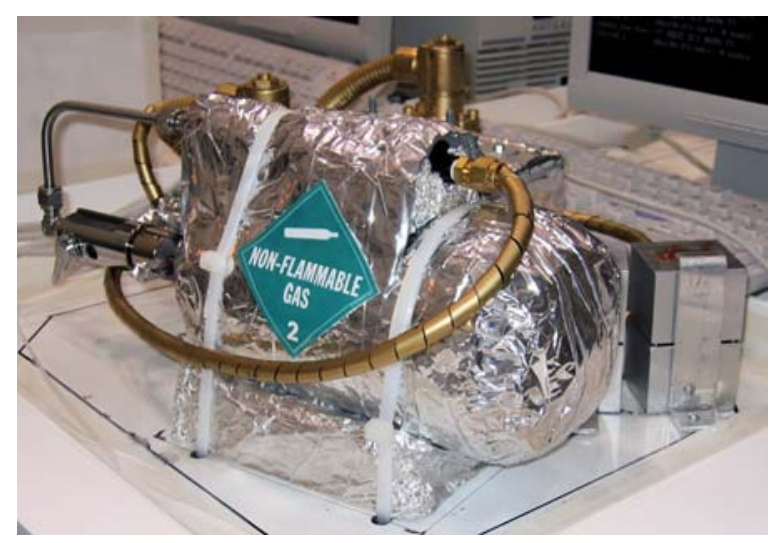

Fig. 13: The LSU Mechanical Engineering rocket nozzle performance student payload. 
control available over the web. The downlinked streaming video is retransmitted over the internet for general viewing. CosmoCam is particularly powerful with HASP as it allows student teams to visually inspect and monitor their payload during flight.

\section{Conclusions}

The High Altitude Student Platform (HASP) was developed to support advanced student-built payloads and to fill the gap in flight capability between latex sounding balloon and satellite launch platforms. The HASP hardware and software is based on the heritage of the LSU ATIC cosmic ray long duration balloon experiment and has included new innovations in modularity to reduce operational cost with increased reliability plus a web portal that provides all flight data in near realtime to students at their home institutions. The first HASP flight was launched on September 4, 2006 at 15:51 UTC and lasted a total of 18 hours and 11 minutes, with 15 of these hours at an average float altitude of 36.3 kilometers. A total of eight payloads from student groups at four institutions were supported during this flight. Topics investigated by the students include rocket nozzle performance, high energy cosmic rays, remote sensing, and studies of the balloon thermal properties and dynamics. Preliminary results indicate that HASP is performing its primary goal of providing students with a learning experience in designing, developing and operating an aerospace experiment. In some cases this learning involves understanding how the space environment requires different design methodologies or how obtaining quantifiable results requires understanding experiment details. In any event, all students benefited greatly from the practical experiences and skills acquired while participating in a HASP flight. The NASA Balloon Program Office has committed to supporting two more flights, and we are currently in the process of soliciting student payloads for HASP 2007. Documentation and details about HASP can be found on the HASP website at http://laspace.lsu.edu/hasp/.

\section{Acknowledgements}

Development and construction of HASP plus first flight operations was supported by the Louisiana Education Quality Support Fund contract LEQSF (2005-07) ENH-TR-12 managed by the Louisiana Board of Regents, the Louisiana Space Consortium (NNG05GH22H), the LSU College of Basic Sciences and the LSU Department of Physics and Astronomy. In addition, the NASA Balloon Program Office plus the Columbia Scientific Balloon Facility provided the personnel, balloon equipment and facility infrastructure used by HASP during testing, prelaunch, flight and recovery operations.

\section{References}

Aldridge, E.C. Jr., Fiorina, C.S., Jackson, M.P., Leshin, L.A., Lyles, L.L., Spudis, P.D., Tyson, N.D., Walker, R.S., and Zuber, M.T., "Report of the President's Commission on Implementation of United State Space Exploration Policy", ISBN 0-16-073075-9, Superintendent of Documents, U.S. Government Printing Office, Mail Stop SSOP, Washington, DC 20402-001, 2004

Ellison, B., Giammanco, J., Guzik, T.G., Johnson, K., and Wefel, J.P., “The Louisiana ACES StudentBuilt BalloonSat Program", Adv. Space Res., in press, 2006

Guzik, T.G. and Wefel, J. P. "The high altitude student platform (HASP) for student-built payloads", $A d v$. Space Res., 37, 2125-2131, 2006

Guzik, T.G., "The Aerospace Catalyst Experiences for Students (ACES) Project”, Suborbital Center of Excellence Annual Student Conference, 2003, http://aspace.lsu.edu/aces/Presentations.html.

Guzik, T.G., Adams, J.H., Ahn, H.S., et al., "The Advanced Thin Ionization Calorimeter (ATIC) for Studies of High Energy Cosmic Rays", Proc. $26^{\text {th }}$ Int. Cosmic Ray Conf. (Salt Lake City), 5, 9-12, 1999

Guzik, T.G., Adams, J.H., Ahn, H.S., et al., "The ATIC Long Duration Balloon Project", Adv. Space Res., $33,1763-1770,2003$ 
National Science Board, "Science and Engineering Indicators 2004", Two volumes, Arlington, VA: National Science Foundation (volume 1, NSB 04-1; volume 2, NSB 04-1A), 2004

Hart, G., Rudman, W. B., Armstrong, A., et al., "Road Map for National Security: Imperative for Change", The Phase III Report of the U.S. Commission on National Security / $21^{\text {st }}$ Century, Washington, D.C., 2001, http://govinfo.library.unt.edu/nssg/index.html

Walker, R.S., Peters, F.W., Aldrin, B., et al., "Final Report of the Commission on the Future of the United States Aerospace Industry", Crystal Gateway 1, Suite 940, 1235 Jefferson Davis Highway, Arlington, Virginia 22202, 2002,

http://ita.doc.gov/td/aerospace/aerospacecommission/aerospacecommission.htm 